\title{
Is leptin protective against heart failure with preserved ejection fraction? A complex interrelationship among leptin, obesity, and left ventricular hypertrophy
}

\author{
Yuichi J. Shimada ${ }^{1}$
}

Received: 4 July 2018 / Revised: 5 September 2018 / Accepted: 5 September 2018 / Published online: 12 November 2018

(c) The Japanese Society of Hypertension 2018

The U.S. has experienced an epidemic of heart failure with preserved ejection fraction (HFpEF), which currently affects at least 3 million American adults [1]. The prognosis after a hospitalization for HFpEF is dismal, with a 5-year survival of 35\%-this is worse than most malignancies [1]. $\mathrm{HFpEF}$ is especially prevalent in African-Americans [2], and this can be attributed to a high prevalence of hypertension and obesity in this population [3]. Thus far, no therapy has been shown to provide mortality benefits in patients with HFpEF. An important knowledge gap exists in the underlying molecular pathways that lead to the development of left ventricular (LV) hypertrophy, stiff LV, and resultant HFpEF. Without a deeper understanding of the pathophysiology through which risk factors of HFpEF lead to adverse LV remodeling and diastolic dysfunction, it is unlikely that primary prevention efforts to avoid HFpEF will succeed.

Leptin is a hormone excreted from adipose tissue (thus its concentration positively correlates with body mass index $[\mathrm{BMI}])$ and is known to have complex effects on the cardiovascular system $[4,5]$. Leptin results in hypertrophy of cardiomyocytes in vitro [6]. In physiological studies in rats, it has been shown that leptin leads to unfavorable neurohormonal changes via activation of the sympathetic nervous system [7]. The resultant elevation in blood pressure may cause LV hypertrophy and HFpEF. However, using a mouse model of obesity, investigators have shown that leptin may be protective against LV hypertrophy $[8,9]$. For instance, obese mice lacking leptin developed LV hypertrophy, which was reversed with the administration of leptin

Yuichi J. Shimada

ys3053@cumc.columbia.edu

1 Division of Cardiology, Department of Medicine, Columbia University Medical Center, New York, NY, USA
[9]. As another example, a mouse model of leptin resistance (i.e., lacking the leptin receptor) showed a reduction in LV hypertrophy only after heart-specific expression of the leptin receptor [8]. Moreover, leptin decreases appetite and increases energy expenditure, both of which can result in weight loss and an improvement in hypertension, sleep apnea, and metabolic derangements such as hyperinsulinemia and lipotoxicity $[10,11]$. Given recent studies reporting the preventive effects of substantial weight loss against the development of new-onset HF and associated morbidities [12, 13], leptin may act as a protective factor against HFpEF through weight reduction. However, the overall effects of leptin with regard to HFpEF in humans are largely unknown.

In this context, the cross-sectional study by Kamimura et al. [14], which was published in the prior issue of Hypertension Research, examined the relationship between plasma leptin concentrations and indices of LV structure and function in 1172 African-Americans with preserved EF using a community-based database. Stratified analyses were performed according to sex and BMI quartiles. Interestingly, leptin concentrations were significantly and inversely associated with LV mass only in women within the 3rd BMI quartile but not in the other subpopulations after adjusting for potentially confounding factors. Similarly, the association between leptin concentrations and diastolic wall strain-an indicator of LV stiffness-was significant only in women within the 4th BMI quartile. These findings are in line with those of the aforementioned studies in obese mice in which a high leptin concentration was protective against LV hypertrophy only in obese subjects [8, 9]. An intriguing hypothesis generated from these findings would be that obese patients with resistance to the weight-reduction effects of leptin have higher leptin concentrations, which in turn exerts direct effects on the heart to prevent the development of hypertrophied and stiff LV. 
These observations need to be interpreted with caution given the eight multiple comparisons (i.e., two sex categories and four BMI quartiles). It should also be noted that the generalizability of these findings is limited to obese African-American women. Additionally, due to the crosssectional study design, the present study does not provide information on the temporal relationship or causality between leptin concentration and the incidence of HFpEF. This study should encourage further research on the molecular basis of the sex-specific and BMI-specific effects of leptin and its predictive value, i.e., whether leptin concentration predicts $\mathrm{HF}$ incidence and/or HF-related morbidities such as acute exacerbation.

In conclusion, the relationships among leptin concentration, BMI, and LV hypertrophy/stiffness are complex and warrant further investigation to determine whether leptin has protective effects against the development of $\mathrm{HFpEF}$.

Funding Dr. Shimada was supported in part by unrestricted grants from the American Heart Association Career Development Award, the American Heart Association National Clinical and Population Research Award, and Honjo International Scholarship Foundation. The researcher was independent from the funding organizations.

\section{Compliance with ethical standards}

Conflict of interest The authors declare that they have no conflict of interest.

\section{References}

1. Research Priorities in Heart Failure with Preserved Ejection Fraction (HFpEF). National Heart, Lung, and Blood Institute, Bethesda, MD. 2017. https://www.nhlbi.nih.gov/events/2017/ research-priorities-heart-failure-preserved-ejection-fraction-hfpef. Accessed 27 August, 2018.

2. Gupta DK, Shah AM, Castagno D, Takeuchi M, Loehr LR, Fox ER, et al. Heart failure with preserved ejection fraction in African Americans: The ARIC (Atherosclerosis Risk In Communities) study. JACC Heart Fail. 2013;1:156-63.
3. Krzesinski P, Stanczyk A, Piotrowicz K, Gielerak G, UziebloZyczkowska B, Skrobowski A. Abdominal obesity and hypertension: a double burden to the heart. Hypertens Res. 2016; 39:349-55.

4. Frederich RC, Hamann A, Anderson S, Lollmann B, Lowell BB, Flier JS. Leptin levels reflect body lipid content in mice: evidence for diet-induced resistance to leptin action. Nat Med. 1995; $1: 1311-4$.

5. Couillard C, Lamarche B, Mauriège P, Cantin B, Dagenais GR, Moorjani S, et al. Leptinemia is not a risk factor for ischemic heart disease in men. Prospective results from the Quebec Cardiovascular Study. Diabetes Care. 1998;21:782-6.

6. Madani S, De Girolamo S, Munoz DM, Li RK, Sweeney G. Direct effects of leptin on size and extracellular matrix components of human pediatric ventricular myocytes. Cardiovasc Res. 2006; 69:716-25.

7. Schwartz MW, Seeley RJ, Campfield LA, Burn P, Baskin DG. Identification of targets of leptin action in rat hypothalamus. J Clin Invest. 1996;98:1101-6.

8. Hall ME, Maready MW, Hall JE, Stec DE. Rescue of cardiac leptin receptors in $\mathrm{db} / \mathrm{db}$ mice prevents myocardial triglyceride accumulation. Am J Physiol Endocrinol Metab. 2014;307: E316-25.

9. Barouch LA, Berkowitz DE, Harrison RW, O'Donnell CP, Hare JM. Disruption of leptin signaling contributes to cardiac hypertrophy independently of body weight in mice. Circulation. 2003; 108:754-9.

10. Alpert MA, Agrawal H, Aggarwal K, Kumar SA, Kumar A. Heart failure and obesity in adults: pathophysiology, clinical manifestations and management. Curr Heart Fail Rep. 2014;11:156-65.

11. Sun SJ, Yao JL, Xu LB, Rui Q, Zhang NN, Chen M, et al. Cardiac structural remodeling in hypertensive cardiomyopathy. Hypertens Res. 2017;40:450-6.

12. Sundstrom J, Bruze G, Ottosson J, Marcus C, Naslund I, Neovius $\mathrm{M}$. Weight loss and heart failure: a nationwide study of gastric bypass surgery versus intensive lifestyle treatment. Circulation. 2017; 135:1577-85.

13. Shimada YJ, Tsugawa Y, Brown DF, Hasegawa K. Bariatric surgery and emergency department visits and hospitalizations for heart failure exacerbation: Population-based, self-controlled series. J Am Coll Cardiol. 2016;67:895-903.

14. Kamimura D, Suzuki T, Wang W, deShazo M, Hall JE, Winniford $\mathrm{MD}$, et al. Higher plasma leptin levels are associated with reduced left ventricular mass and left ventricular diastolic stiffness in black women: insights from the Genetic Epidemiology Network of Arteriopathy (GENOA) study. Hypertens Res. 2018. https://doi. org/10.1038/s41440-018-0062-0. 